\title{
A Discrete-Time Queue with Balking, Reneging, and Working Vacations
}

\author{
Veena Goswami \\ School of Computer Application, KIIT University, Bhubaneswar 751024, India \\ Correspondence should be addressed to Veena Goswami; veena_goswami@yahoo.com
}

Received 21 May 2014; Accepted 9 October 2014; Published 29 October 2014

Academic Editor: Onesimo Hernandez-Lerma

Copyright (C) 2014 Veena Goswami. This is an open access article distributed under the Creative Commons Attribution License, which permits unrestricted use, distribution, and reproduction in any medium, provided the original work is properly cited.

\begin{abstract}
This paper presents an analysis of balking and reneging in finite-buffer discrete-time single server queue with single and multiple working vacations. An arriving customer may balk with a probability or renege after joining according to a geometric distribution. The server works with different service rates rather than completely stopping the service during a vacation period. The service times during a busy period, vacation period, and vacation times are assumed to be geometrically distributed. We find the explicit expressions for the stationary state probabilities. Various system performance measures and a cost model to determine the optimal service rates are presented. Moreover, some queueing models presented in the literature are derived as special cases of our model. Finally, the influence of various parameters on the performance characteristics is shown numerically.
\end{abstract}

\section{Introduction}

Discrete-time queueing models with server vacations have been studied extensively in recent years by many researchers because these systems are better suited than their continuoustime counterparts to analyze and design digital transmitting systems. Extensive analysis of a wide variety of discrete-time queueing models with vacations has been reported in Takagi [1]. Queues with balking and reneging often arise in practice, when there is a tendency of customers to be impatient due to a long queue. As a result, the customers either balk (i.e., decide not to join the queue) or renege (i.e., leave after joining the queue without getting served). The significance of this system emerges in many real life situations such as telephone services, computer and communication systems, production line systems, machine operating systems, inventory systems, and the hospital emergency rooms.

In the study of working vacation models, the server remains active during the vacation period and serves the customers generally at a slower rate. At a vacation termination epoch, if the queue is nonempty, a regular service period begins with normal service rate; otherwise the server takes another vacation. This vacation policy is called multiple working vacations (MWV). In the single working vacation
(SWV) policy, the server takes only one working vacation when the system becomes empty. When the server returns from a SWV, it stays in the system waiting for customers to arrive instead of taking another working vacation. Otherwise, it alters the service rate back to the normal service rate as in the MWV policy. An extensive report of a wide variety of working vacation queueing models can be found in Servi and Finn [2], Wu and Takagi [3], Tian et al. [4], Li and Tian [5], and the references therein.

Impatience is the most important feature in queueing systems, as individuals always feel concerned while waiting for service. Performance analysis of queueing systems with balking and reneging in real life congestion problems is beneficial because one finds new managerial insights. While making decision for the service rate of servers needed in the service system to meet time-varying demand, the balking and reneging probabilities can be used to estimate the amount of lost revenues as reported in Liao [6]. The study of an $M / M / 1 / N$ queue with balking, reneging, and multiple server vacations has been studied in Yue et al. [7]. The optimal balking strategies in a Markovian queue with a single exponential vacation have been discussed in Tian and Yue [8]. Ma et al. [9] examined an equilibrium balking behavior in the Geo/Geo/1 queueing system with multiple 
vacations. Zhang et al. [10] have investigated the equilibrium balking strategies for observable and unobservable $M / M / 1$ queue with working vacations. Laxmi et al. [11] analyzed an $M / M / 1 / N$ queueing system with balking, reneging, and working vacations. Laxmi et al. [12] studied an optimization of balking and reneging queue with vacation interruption under $N$-policy.

The study of the discrete-time queueing systems with impatient customers' behavior and vacations seems to be a recent endeavor. A discrete-time single server queue with balking has been examined by Lozano and Moreno [13]. Vijaya Laxmi et al. [14] have considered a Geo/Geo/1/N queue with balking and multiple working vacations. Queueing models with balking, reneging, and server working vacations accommodate the real world situations more closely from an economic viewpoint. However, most of the research works in discrete-time queueing systems have not yet considered balking and reneging with server working vacations simultaneously. It appears to be more pragmatic that the balking and reneging behaviors are studied with working vacations in the discrete-time queuing models.

This paper focuses on a finite buffer balking and reneging in discrete-time single server queue with single and multiple working vacations. The interarrival times of customers and service times are assumed to be independent and geometrically distributed. The service times during a vacation period and vacation times are assumed to be geometrically distributed. The customers are allowed to decide whether to join or balk or renege, that is, leave after joining the queue without getting served. We present a recursive method to obtain the stationary-state probabilities for late arrival system with delayed access. Various performance measures have been discussed. Numerical results in the form of tables and graphs are presented to display the impact of the system parameters on the performance measures. We use quadratic fit search method to minimize the total expected cost per unit time. The proposed cost model may be useful to system engineers in finding the optimal service rate desired for operating the system smoothly at optimum cost. This model has potential applications in many congestion situations of communication systems, manufacturing systems, production/inventory systems, transportation, and many others.

This paper is structured as follows. In Section 2, we present the description of the queueing model. The analysis of the model for the stationary-state probabilities is given in Section 3. Performance measures and cost analysis are carried out in Section 4. Some special cases which are matched with existing results in the literature are demonstrated in Section 5. Numerical results in the form of tables and graphs are presented in Section 6. Section 7 concludes the paper.

\section{Model Description}

We consider a finite buffer discrete-time balking and reneging single server queue with single and multiple working vacations under the late arrival system with delayed access (LASDA). Let us assume that the time axis is slotted into intervals of equal length with the length of a slot being unity, and it is marked as $0,1,2, \ldots, t, \ldots$ Here, a potential arrival takes place in $(t-, t)$ and a potential departure occurs in $(t, t+)$. More specifics on discrete-time queue have been reported in Hunter [15] and Gravey and Hébuterne [16].

We assume that the interarrival times $A$ of customers are independent and geometrically distributed with probability mass function (p.m.f.) $a_{n}=P(A=n)=\bar{\lambda}^{n-1} \lambda, n \geq 1,0<$ $\lambda<1$, where for any real number $x \in[0,1]$, we denote $\bar{x}=1-x$. If on arrival customer finds the system busy, the arriving customer either decides to join the queue or balk. Let $b_{n}$ represent the probability, when the system size is $n$ with which the customer decides to join the queue in order to be served or balk with probability $1-b_{n}$, when there are $n$ customers in the system upon arrival $(n=0,1, \ldots, N-1)$, where the capacity of the system is finite $N$. Furthermore, we assume that $0 \leq b_{n+1} \leq b_{n}<1,1 \leq n \leq N-1, b_{0}=1, b_{N}=0$.

The customers are served on a first-come, first-served (FCFS) discipline. Once service commences it always proceeds to completion. The service times of customers are independent and geometrically distributed with probability mass function (p.m.f.): $P(S=n)=\bar{\mu}^{n-1} \mu, n \geq 1,0<$ $\mu<1$. The service times $S_{v}$ in a working vacation period are independent and geometrically distributed with common p.m.f.: $P\left(S_{v}=n\right)=\bar{\eta}^{n-1} \eta, n \geq 1,0<\eta<1$. The server commences multiple working vacations on the instant when the system becomes empty. If the system is empty when the server returns from a working vacation, it begins another working vacation. Otherwise, the server changes to a service period and a regular busy period begins. Under the single working vacation, when the system becomes empty, the server takes only one working vacation. It remains in the system waiting for customers to arrive rather than taking another working vacation. Otherwise, it switches the service rate back to the busy period as under the MWV policy. The vacation times $V$ are independent and geometrically distributed with common p.m.f.: $P(V=n)=\bar{\phi}^{n-1} \phi, n \geq 1$, $0<\phi<1$.

After joining the queue each customer will wait a certain length of time $T$ for service to begin. If it has not begun by then, he will get impatient and leave the queue without getting service. This time $T$ is independent and geometrically distributed with common p.m.f.: $P(T=n)=\bar{\alpha}^{n-1} \alpha, n \geq 1$, $0<\alpha<1$. Since the arrival and the departure of the impatient customers without service are independent, the average reneging rate of the customer can be given by $r(n)=$ $(n-1) \alpha, 1 \leq n \leq N$.

\section{Analysis of the Model}

In this section, we present the analytic analysis of finite buffer discrete-time balking and reneging single server queue with single and multiple working vacations. We analyze both multiple working vacations and single working vacation models concurrently. For that reason we introduce an indicator function $(\delta)$ as follows: $\delta=1$ yields the results for the SWV model and $\delta=0$ gives the results for the MWV model. 
At steady-state, let us define $\pi_{i, 0}, 0 \leq i \leq N$, to be the probability that there are $i$ customers in the system when the server is in working vacation and $\pi_{i, 1}, 1-\delta \leq i \leq N$, to be the probability that there are $i$ customers in the system when the server is in regular busy period.

Based on the one-step transition analysis, the steady-state equations can be written as

$$
\begin{aligned}
& \pi_{0,0}=\bar{\lambda}(1-\phi \delta) \pi_{0,0}+\mathbf{s}_{1}(\eta) \pi_{1,0} \\
& +\mathbf{t}_{2}(\eta) \pi_{2,0}+\mathbf{s}_{1}(\mu) \pi_{1,1}+\mathbf{t}_{2}(\mu) \pi_{2,1}, \\
& \pi_{i, 0}=\bar{\phi} \mathbf{r}_{i}(\eta) \pi_{i, 0}+\bar{\phi} \mathbf{q}_{i-1}(\eta) \pi_{i-1,0}+\bar{\phi} \mathbf{s}_{i+1}(\eta) \pi_{i+1,0} \\
& +\bar{\phi} \mathbf{t}_{i+2}(\eta) \pi_{i+2,0}, \quad 1 \leq i \leq N-2, \\
& \pi_{N-1,0}=\bar{\phi} \mathbf{r}_{N-1}(\eta) \pi_{N-1,0}+\bar{\phi} \mathbf{q}_{N-2}(\eta) \pi_{N-2,0} \\
& +\bar{\phi} \mathbf{s}_{N}(\eta) \pi_{N, 0} \text {, } \\
& \pi_{N, 0}=\bar{\phi} \mathbf{r}_{N}(\eta) \pi_{N, 0}+\bar{\phi} \mathbf{q}_{N-1}(\eta) \pi_{N-1,0}, \\
& \pi_{0,1}=\bar{\lambda} \pi_{0,1}+\phi \bar{\lambda} \delta \pi_{0,0}, \\
& \pi_{1,1}=\mathbf{r}_{1}(\mu) \pi_{1,1}+\mathbf{s}_{2}(\mu) \pi_{2,1}+\mathbf{t}_{3}(\mu) \pi_{3,1} \\
& +\mathbf{q}_{0}(\mu) \delta \pi_{0,1}+\phi \mathbf{r}_{1}(\eta) \pi_{1,0}+\phi \mathbf{q}_{0}(\eta) \pi_{0,0} \\
& +\phi \mathbf{s}_{2}(\eta) \pi_{2,0}+\bar{\phi} \mathbf{t}_{3}(\eta) \pi_{3,0}, \\
& \pi_{i, 1}=\mathbf{r}_{i}(\mu) \pi_{i, 1}+\mathbf{q}_{i-1}(\mu) \pi_{i-1,1}+\mathbf{s}_{i+1}(\mu) \pi_{i+1,1} \\
& +\mathbf{t}_{i+2}(\mu) \pi_{i+2,1}+\phi \mathbf{r}_{i}(\eta) \pi_{i, 0}+\phi \mathbf{q}_{i-1}(\eta) \pi_{i-1,0} \\
& +\phi \mathbf{s}_{i+1}(\eta) \pi_{i+1,0}+\phi \mathbf{t}_{i+2}(\eta) \pi_{i+2,0}, \\
& 1 \leq i \leq N-2, \\
& \pi_{N-1,1}=\mathbf{r}_{N-1}(\mu) \pi_{N-1,1}+\mathbf{q}_{N-2}(\mu) \pi_{N-2,1} \\
& +\mathbf{s}_{N}(\mu) \pi_{N, 1}+\phi \mathbf{r}_{N-1}(\eta) \pi_{N-1,0} \\
& +\phi \mathbf{q}_{N-2}(\eta) \pi_{N-2,0}+\phi \mathbf{s}_{N}(\eta) \pi_{N, 0}, \\
& \pi_{N, 1}=\mathbf{r}_{N}(\mu) \pi_{N, 1}+\mathbf{q}_{N-1}(\mu) \pi_{N-1,1} \\
& +\phi \mathbf{r}_{N}(\eta) \pi_{N, 0}+\phi \mathbf{q}_{N-1}(\eta) \pi_{N-1,0},
\end{aligned}
$$

where

$$
\begin{aligned}
& \mathbf{q}_{i}(x)= \begin{cases}\lambda: & i=0, \\
\lambda b_{i} \bar{x}(1-(i-1) \alpha): & i=1, \ldots, N-1,\end{cases} \\
& \mathbf{r}_{i}(x)=\left\{\begin{array}{cl}
\left(1-\lambda b_{1}\right) \bar{x}+\lambda b_{1} x: & i=1, \\
\left(1-\lambda b_{i}\right) \bar{x}(1-(i-1) \alpha) & \\
+\lambda b_{i}(x(1-(i-1) \alpha) & \\
+\bar{x}(i-1) \alpha): & i=2, \ldots, N-1, \\
\bar{x}(1-(N-1) \alpha): & i=N,
\end{array}\right.
\end{aligned}
$$

$\mathbf{s}_{i}(x)$

$$
\begin{aligned}
& =\left\{\begin{aligned}
\left(1-\lambda b_{1}\right) x: & i=1, \\
\left(1-\lambda b_{i}\right)(x(1-(i-1) \alpha) & \\
+\bar{x}(i-1) \alpha) & \\
+\lambda b_{i} x(i-1) \alpha: & i=2, \ldots, N-1, \\
x(1-(N-1) \alpha)+\bar{x}(N-1) \alpha: & i=N,
\end{aligned}\right. \\
& \mathbf{t}_{i}(x)= \begin{cases}\left(1-\lambda b_{i}\right) x(i-1) \alpha: & i=2, \ldots, N-1, \\
x(N-1) \alpha: & i=N .\end{cases}
\end{aligned}
$$

The steady-state probabilities $\pi_{i, 0}(0 \leq i \leq N)$ and $\pi_{i, 1},(1-$ $\delta \leq i \leq N$ ) are computed recursively by solving the system of (1) to (9). Solving (2) to (4) recursively, it can be simplified as

$$
\pi_{i, 0}=\xi_{i} \pi_{N, 0}, \quad 0 \leq i \leq N,
$$

where

$$
\begin{aligned}
\xi_{N} & =1, \quad \xi_{N-1}=\left(\frac{1-\bar{\phi} \mathbf{r}_{N}(\eta)}{\bar{\phi} \mathbf{q}_{N-1}(\eta)}\right) \xi_{N}, \\
\xi_{N-2} & =\frac{\left(1-\bar{\phi} \mathbf{r}_{N-1}(\eta)\right) \xi_{N-1}-\bar{\phi} \mathbf{s}_{N}(\eta) \xi_{N}}{\bar{\phi} \mathbf{q}_{N-2}(\eta)}, \\
\xi_{i} & =\frac{\left(1-\bar{\phi} \mathbf{r}_{i+1}(\eta)\right) \xi_{i+1}-\bar{\phi} \mathbf{s}_{i+2}(\eta) \xi_{i+2}-\bar{\phi} \mathbf{t}_{i+3}(\eta) \xi_{i+3}}{\bar{\phi} \mathbf{q}_{i}(\eta)}, \\
i=N-3, \ldots, 1,0 . &
\end{aligned}
$$

Using (11) in (5) and (7)-(9) yields $\pi_{i, 1},(1-\delta \leq i \leq N)$, in terms of $\pi_{N, 0}$ and $\pi_{N, 1}$ as

$$
\pi_{i, 1}=\zeta_{i} \pi_{N, 1}+\omega_{i} \pi_{N, 0}, \quad 0 \leq i \leq N,
$$

where $\zeta_{N}=1, \omega_{N}=0$,

$$
\begin{gathered}
\zeta_{N-1}=\frac{1-\mathbf{r}_{N}(\mu)}{\mathbf{q}_{N-1}(\mu)} \\
\omega_{N-1}=-\phi\left(\frac{\mathbf{r}_{N}(\eta) \xi_{N}+\mathbf{q}_{N-1}(\eta) \xi_{N-1}}{\mathbf{q}_{N-1}(\mu)}\right), \\
\zeta_{N-2}=\frac{\left(1-\mathbf{r}_{N-1}(\mu)\right) \zeta_{N-1}-\mathbf{s}_{N}(\mu) \zeta_{N}}{\mathbf{q}_{N-2}(\mu)},
\end{gathered}
$$

$\omega_{N-2}$

$$
\begin{aligned}
&=\left(\left(1-\mathbf{r}_{N-1}(\mu)\right) \omega_{N-1}\right. \\
&\left.-\phi\left(\mathbf{q}_{N-2}(\eta) \xi_{N-2}+\mathbf{r}_{N-1}(\eta) \xi_{N-1}+\mathbf{s}_{N}(\eta) \xi_{N}\right)\right) \\
& \times\left(\mathbf{q}_{N-2}(\mu)\right)^{-1} \\
& \zeta_{i-1}=\frac{\left(1-\mathbf{r}_{i}(\mu)\right) \zeta_{i}-\mathbf{s}_{i+1}(\mu) \zeta_{i+1}-\mathbf{t}_{i+2}(\mu) \zeta_{i+2}}{\mathbf{q}_{i-1}(\mu)}, \\
& i=N-2, \ldots, 1,
\end{aligned}
$$




$$
\begin{gathered}
\omega_{i-1}=\frac{\left(1-\mathbf{r}_{i}(\mu)\right) \omega_{i}-\mathbf{s}_{i+1}(\mu) \omega_{i+1}-\mathbf{t}_{i+2}(\mu) \omega_{i+2}}{\mathbf{q}_{i-1}(\mu)} \\
-\phi\left(\left(\mathbf{r}_{i}(\eta) \xi_{i}+\mathbf{q}_{i-1}(\eta) \xi_{i-1}\right.\right. \\
\left.\quad+\mathbf{s}_{i+1}(\eta) \xi_{i+1}+\mathbf{t}_{i+2}(\eta) \xi_{i+2}\right) \\
\left.\quad \times\left(\mathbf{q}_{i-1}(\mu)\right)^{-1}\right), \quad i=N-2, \ldots, 2, \\
\zeta_{0}=0, \quad \omega_{0}=\left(\frac{\phi \bar{\lambda} \delta \xi_{0}}{\lambda}\right) .
\end{gathered}
$$

Now, using (11) and (13) in (6), the probability $\pi_{N, 1}$ can be expressed in terms of $\pi_{N, 0}$ as

$$
\pi_{N, 1}=\Upsilon \pi_{N, 0},
$$

where

$\Upsilon$

$$
\begin{aligned}
= & \phi\left(\frac{\mathbf{r}_{1}(\eta) \xi_{1}+\mathbf{q}_{0}(\eta) \xi_{0}+\mathbf{s}_{2}(\eta) \xi_{2}+\mathbf{t}_{3}(\eta) \xi_{3}}{\left(1-\mathbf{r}_{1}(\mu)\right) \zeta_{1}-\mathbf{s}_{2}(\mu) \zeta_{2}-\mathbf{t}_{3}(\mu) \zeta_{3}}\right) \\
& +\left(\frac{\mathbf{s}_{2}(\mu) \omega_{2}+\mathbf{t}_{3}(\mu) \omega_{3}+\delta \mathbf{q}_{0}(\mu) \omega_{0}-\left(1-\mathbf{r}_{1}(\mu)\right) \omega_{1}}{\left(1-\mathbf{r}_{1}(\mu)\right) \zeta_{1}-\mathbf{s}_{2}(\mu) \zeta_{2}-\mathbf{t}_{3}(\mu) \zeta_{3}}\right) .
\end{aligned}
$$

Finally, the only unknown $\pi_{N, 0}$ is obtained from the normalization condition $\sum_{i=0}^{N} \pi_{N, 0}+\sum_{i=1-\delta}^{N} \pi_{N, 1}=1$ and is given by

$$
\pi_{N, 0}=\left[\sum_{i=0}^{N} \xi_{i}+\sum_{i=1-\delta}^{N}\left(\omega_{i}+\Upsilon \zeta_{i}\right)\right]^{-1} .
$$

This completes the evaluation of steady-state probabilities.

\section{Performance Measures}

As the system length distributions at various epochs are known, performance measures such as average number of customers in the system (queue) $L_{s}\left(L_{q}\right)$ are given by

$$
\begin{aligned}
L_{s} & =\sum_{i=1}^{N} i \pi_{i, 0}+\sum_{i=1}^{N} i \pi_{i, 1}, \\
L_{q} & =\sum_{i=1}^{N}(i-1) \pi_{i, 0}+\sum_{i=1}^{N}(i-1) \pi_{i, 1} .
\end{aligned}
$$

The busy probability of the server $\left(P_{B}\right)$, probability that the server is in working vacation $\left(P_{\mathrm{wv}}\right)$, and probability that the server is idle $\left(P_{\text {id }}\right)$ are given as

$$
P_{B}=\sum_{i=1}^{N} \pi_{i, 1}, \quad P_{\mathrm{wv}}=\sum_{i=0}^{N} \pi_{i, 0}, \quad P_{\mathrm{id}}=\delta \pi_{0,1} .
$$

When there are $i$ customers in the system upon arrival, the probability that a customer balks from the system is $1-b_{i}$, and the immediate balking rate is $\lambda\left(1-b_{i}\right)$. The average balking rate (B.R.) is given by

$$
\text { B.R. }=\sum_{i=1}^{N} \lambda\left(1-b_{i}\right) \pi_{i, 0}+\sum_{i=1}^{N} \lambda\left(1-b_{i}\right) \pi_{i, 1} .
$$

If there are $i$ customers in the system and server is available, then there are $(i-1)$ waiting customers in the queue. Since any one of the $(i-1)$ customers in the queue may renege, the instantaneous reneging rate is $(i-1) \alpha$. The average reneging rate (R.R.) is given by

$$
\text { R.R. }=\sum_{i=1}^{N}(i-1) \alpha \pi_{i, 0}+\sum_{i=1}^{N}(i-1) \alpha \pi_{i, 1} .
$$

Finally, the average rate of customer loss (L.R.) is the sum of the average balking rate and the average reneging rate and yields

$$
\text { L.R. }=\text { B.R. }+ \text { R.R. }
$$

4.1. Cost Model. In this subsection, we develop an expected cost function to determine an optimum regular service rate, $\mu^{*}$, and the optimum total expected cost, $F\left(\mu^{*}\right)$. Let us define the following cost elements:

$C_{1} \equiv$ service cost per unit time when the server is in regular busy period,

$C_{2} \equiv$ service cost per unit time when the server is on working vacation period,

$C_{3} \equiv$ cost per unit time when a customer joins the queue and waits for service,

$C_{4} \equiv$ cost per unit time when a customer balks or reneges.

Based on the definitions of each cost element listed above and its corresponding system performance measures, the total expected cost function per unit time is given by

$$
F(\mu)=C_{1} \mu+C_{2} \eta+C_{3} L_{q}+C_{4} \text { L.R., }
$$

where $L_{q}$ and L.R. are given in the previous section. The first two costs are obtained by the server, the third one by the customer's waiting in the queue, and the fourth one by the customer loss. The objective is to determine the optimal service rate $\mu^{*}$ to minimize the cost function $F$. As the expected cost function is highly complex, it is a difficult task to develop analytic results for the optimum value of $\mu$. We use the quadratic fit search method to solve the above optimization problem as given in Rardin [17]. Given a 3-point pattern, the unique optimum $x$ of the quadratic function agreeing with $f(x)$ at $\left(x_{0}, x_{1}, x_{2}\right)$ occurs at

$x$

$$
=\frac{1}{2} \frac{f\left(x_{0}\right)\left(x_{1}^{2}-x_{2}^{2}\right)+f\left(x_{1}\right)\left(x_{2}^{2}-x_{0}^{2}\right)+f\left(x_{2}\right)\left(x_{0}^{2}-x_{1}^{2}\right)}{f\left(x_{0}\right)\left(x_{1}-x_{2}\right)+f\left(x_{1}\right)\left(x_{2}-x_{0}\right)+f\left(x_{2}\right)\left(x_{0}-x_{1}\right)} .
$$




\section{Special Cases}

In this section, some special cases which are available in the literature are deduced from our model by taking specific values for the parameters $\phi, \eta, b_{i}$, and $\alpha$.

Case 1. Consider $\alpha \rightarrow 0$. That is, the customers never renege. The model reduces to $\mathrm{Geo} / \mathrm{Geo} / 1 / N$ queue with balking and multiple working vacations (for $\delta=0$ ) and single working vacation (for $\delta=1$ ). For $\delta=0$, our results match the results of $\mathrm{Geo} / \mathrm{Geo} / 1 / \mathrm{N}$ queue with balking and multiple working vacations of [14].

Case 2. Consider $\eta \rightarrow 0$. That is, there is no service during vacation. The model reduces to $\mathrm{Geo} / \mathrm{Geo} / 1 / N$ queue with balking, reneging, and multiple vacations (for $\delta=0$ ) and single vacation (for $\delta=1$ ).

Case 3. $\alpha \rightarrow 0, b_{i}=1,0 \leq i \leq N-1$; that is, the customers neither balk nor renege. The model reduces to $\mathrm{Geo} / \mathrm{Geo} / 1 / N$ queue with working vacations. The steady-state probabilities are

$$
\begin{aligned}
& \pi_{i, 0}=\xi_{i} \pi_{N, 0}, \quad 0 \leq i \leq N, \\
& \pi_{i, 1}=\zeta_{i} \pi_{N, 1}+\omega_{i} \pi_{N, 0}, \quad 0 \leq i \leq N,
\end{aligned}
$$

where $\mathbf{q}_{0}(x)=\lambda, \mathbf{q}_{i}(x)=\lambda \bar{x}, 1 \leq i \leq N-1, \mathbf{r}_{i}(x)=\bar{\lambda} \bar{x}+\lambda x$, $1 \leq i \leq N-1, \mathbf{r}_{N}(\mu)=\bar{x}, \mathbf{s}_{i}(x)=\bar{\lambda} x, 1 \leq i \leq N-1, \mathbf{s}_{N}(x)=x$, and $\mathbf{t}_{i}(x)=0 \forall i$.

Case 4. Consider $\eta \rightarrow 0, \phi \rightarrow 1$. That is, the server never takes any vacation. The model reduces to $\mathrm{Geo} / \mathrm{Geo} / 1 / \mathrm{N}$ queue with balking and reneging. In this case the vacation probabilities $\left(\pi_{i, 0}, 1 \leq i \leq N\right)$ do not exist. If we take $\pi_{0,0}$ as $\pi_{0}$ and $\pi_{i, 1}$ as $\pi_{i}$ for $1 \leq i \leq N$, we get the results of balking and reneging $\mathrm{Geo} / \mathrm{Geo} / 1 / N$ queue without vacations.

Case 5. Consider $\eta \rightarrow 0, \phi \rightarrow 1, \alpha \rightarrow 0$. That is, the server never takes any vacation. The model reduces to Geo/Geo/1/N queue with balking and without vacation. To obtain relations steady-state probabilities, let us define $\pi_{0,0}=\pi_{0}$ and $\pi_{i, 1}=\pi_{i}$ for $1 \leq i \leq N$. In this case, the vacation probabilities $\left(\pi_{i, 0}\right.$, $1 \leq i \leq N)$ do not exist. Taking $\mathbf{q}_{1}(\mu)=\lambda, \mathbf{q}_{i}(\mu)=\lambda b_{i} \bar{\mu}, 1 \leq$ $i \leq N-1, \mathbf{r}_{i}(\mu)=\left(1-\lambda b_{i}\right) \bar{\mu}+\lambda b_{i} \mu, 1 \leq i \leq N-1, \mathbf{r}_{N}(\mu)=\bar{\mu}$, $\mathbf{s}_{i}(\mu)=\left(1-\lambda b_{i}\right) \mu, 1 \leq i \leq N-1, \mathbf{s}_{N}(\mu)=\mu$, and $\mathbf{t}_{i}(\mu)=0 \forall i$, we get steady-state probabilities after simplification as

$$
\begin{aligned}
\pi_{i} & =\zeta_{i} \pi_{N}, \quad 0 \leq i \leq N \\
\zeta_{i} & =\frac{\mu \zeta_{N}}{\lambda b_{N-1} \bar{\mu}} \prod_{j=i}^{N-2} \frac{\mu\left(1-\lambda b_{j+1}\right)}{\lambda b_{j} \bar{\mu}}, \\
\zeta_{N} & =\left[1+\frac{\mu}{\lambda b_{N-1} \bar{\mu}} \sum_{i=0}^{N-1} \prod_{j=i}^{N-2} \frac{\mu\left(1-\lambda b_{j+1}\right)}{\lambda b_{j} \bar{\mu}}\right]^{-1} .
\end{aligned}
$$

Our results match analytically the results posed in [13].

Case 6. Consider $\eta \rightarrow 0, \phi \rightarrow 1, \alpha \rightarrow 0$, and $b_{i}=1,0 \leq$ $i \leq N-1$. The model reduces to $\mathrm{Geo} / \mathrm{Geo} / 1 / N$ queue without balking, reneging, and vacation and our results match the results available in the literature. It is to be noted that $\pi_{i, 0}$, $1 \leq i \leq N$, will not occur in this case.

\section{Numerical Analysis}

In this section, we present a variety of numerical results to analyze the operating and economic performance measures for MWV and SWV policies. The balking function is taken as $b_{n}=1-\left(n / N^{2}\right), 1 \leq n \leq N-1, b_{0}=1, b_{N}=0$, and the various cost elements are $C_{1}=25, C_{2}=20, C_{3}=21$, and $C_{4}=18$. We fix the capacity of the system as $N=$ 30. Table 1 shows the minimum cost $F\left(\mu^{*}\right)$, the optimum value of $\mu^{*}$, and some performance measures for MWV and SWV policies. It demonstrates a numerical analysis of some performance measures such as $L_{q}, L_{s}$, L.R., $P_{B}, P_{\mathrm{wv}}$, and $F(\mu)$ for different values of reneging rate $(\alpha)$. The parameters are taken as $\lambda=0.2, \phi=0.4, \eta=0.2, N=10$, and $b_{i}=$ $1-e^{-i}$. We observe that as $\alpha$ increases (i) the optimum $\mu^{*}$ decreases; (ii) the optimum cost and the $P_{\mathrm{wv}}$ decrease for both the models; (iii) the other performance indices increase for both the models; (iv) the single working vacation model has better performance measures than the multiple working vacations model.

We demonstrate the minimum cost $F\left(\mu^{*}\right)$, the optimum value of $\mu^{*}$, and the variations in the system performance measures for MWV and SWV policies in Table 2. It displays comparative numerical results of some performance measures such as $L_{q}, L_{s}$, L.R., $P_{B}, P_{\mathrm{wv}}$, and $F(\mu)$ for different values of service rate during vacation $(\eta)$. The parameters are taken as $\lambda=0.2, \phi=0.4, \alpha=0.01, N=10$, and $b_{i}=1-i / N^{2}$. It can be seen that as $\eta$ increases, (i) the optimum $\mu^{*}$ decreases; (ii) the optimum cost and the $P_{\mathrm{wv}}$ increase for both the models; (iii) the other performance indices decrease for both the models; (iv) the single working vacation model has better performance measures than the multiple working vacations model.

Figure 1 illustrates the effect of service rate during vacation $\eta$ on the average rate of customer loss (L.R.) for various vacation parameters $\phi$ with MWV policy. The parameters are taken as $\lambda=0.2, \mu=0.5, N=10$, and $\alpha=0.1$. We observe that the average rate of customer loss in the system decreases as service rate during vacation increases. When $\mu>\eta$, L.R. decreases with the increase of vacation rate $(\phi)$, but when $\mu<\eta$, L.R. increases with the increase of vacation rate. Hence, a better performance result can be achieved by choosing the value of $\eta$ less than $\mu$, which is coherent with the intuition that the more frequently customers balk, the more customers are in queue and the more possibility to lose customers.

Figure 2 shows the effect of traffic intensity $(\rho)$ on the average queue length $\left(L_{q}\right)$ for different service rates during vacation period $\eta$. The parameters are taken as $\lambda=0.2, \mu=$ $0.7, N=10, \phi=0.1$, and $\alpha=0.05$. We observe that the expected queue length $L_{q}$ monotonically increases as traffic intensity $(\rho)$ increases for both MWV and SWV models. When $\rho$ is kept fixed, $L_{q}$ decreases with the increase of $\eta$. Furthermore, MWV policy outperforms the SWV model. 
TABLE 1: Effect of $\alpha$ on performance characteristics for MWV and SWV models.

\begin{tabular}{lcccccrrr}
\hline & \multicolumn{2}{c}{$\alpha=0.03$} & \multicolumn{2}{c}{$\alpha=0.05$} & \multicolumn{2}{c}{$\alpha=0.07$} & \multicolumn{2}{c}{$\alpha=0.1$} \\
& MWV & SWV & MWV & SWV & MWV & SWV & MWV & SWV \\
\hline$\mu^{*}$ & 0.3648 & 0.3683 & 0.3381 & 0.3439 & 0.3133 & 0.3215 & 0.2807 \\
$L_{q}$ & 0.2169 & 0.1879 & 0.2207 & 0.1933 & 0.2241 & 0.1976 & 0.2263 \\
$L_{s}$ & 0.7214 & 0.6618 & 0.7386 & 0.6827 & 0.7553 & 0.7018 & 0.7760 & 0.2903 \\
$P_{B}$ & 0.3840 & 0.4248 & 0.4008 & 0.4417 & 0.4174 & 0.4579 & 0.4405 & 0.4812 \\
$P_{\text {wV }}$ & 0.6160 & 0.2515 & 0.5992 & 0.2441 & 0.5826 & 0.2370 & 0.5595 \\
L.R. & 0.0358 & 0.0337 & 0.0411 & 0.0386 & 0.0465 & 0.0435 & 0.0545 & 0.2268 \\
$F\left(\mu^{*}\right)$ & 17.5619 & 17.0943 & 17.0414 & 16.6545 & 16.5628 & 16.2478 & 15.9083 & 15.6871 \\
\hline
\end{tabular}

TABLE 2: Effect of $\eta$ on performance characteristics for MWV and SWV models.

\begin{tabular}{|c|c|c|c|c|c|c|c|c|}
\hline & \multicolumn{2}{|c|}{$\eta=0.05$} & \multicolumn{2}{|c|}{$\eta=0.1$} & \multicolumn{2}{|c|}{$\eta=0.2$} & \multicolumn{2}{|c|}{$\eta=0.3$} \\
\hline & MWV & SWV & MWV & SWV & MWV & SWV & MWV & SWV \\
\hline$\mu^{*}$ & 0.4506 & 0.4463 & 0.4475 & 0.4448 & 0.4402 & 0.4422 & 0.4341 & 0.4397 \\
\hline$L_{q}$ & 0.3049 & 0.2376 & 0.2876 & 0.2293 & 0.2591 & 0.2152 & 0.2346 & 0.2046 \\
\hline$L_{s}$ & 0.8532 & 0.7270 & 0.8230 & 0.7127 & 0.7706 & 0.6873 & 0.7228 & 0.6668 \\
\hline$P_{B}$ & 0.4189 & 0.4332 & 0.4084 & 0.4291 & 0.3895 & 0.4214 & 0.3712 & 0.4149 \\
\hline$P_{\mathrm{wv}}$ & 0.5811 & 0.2526 & 0.5916 & 0.2530 & 0.6105 & 0.2537 & 0.6288 & 0.2541 \\
\hline L.R. & 0.0048 & 0.0038 & 0.0045 & 0.0037 & 0.0041 & 0.0035 & 0.0038 & 0.0034 \\
\hline$F\left(\mu^{*}\right)$ & 17.8250 & 16.4920 & 18.4318 & 17.3027 & 19.7305 & 18.9820 & 21.1296 & 20.7263 \\
\hline
\end{tabular}

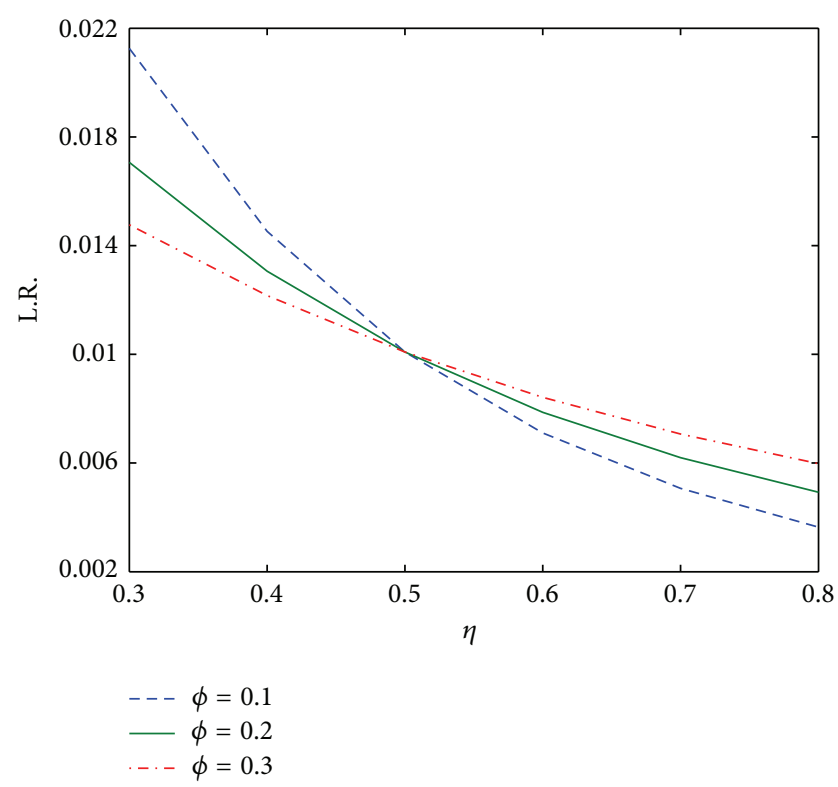

FIGURE 1: Impact of $\eta$ on L.R.

Figure 3 plots the impact of $\eta$ on the state probabilities of the server for various values of vacation parameter $\phi$ with SWV policy. It is observed that as $\eta$ increases, the probability that the server is busy $\left(P_{B}\right)$ decreases, the probability that the server is in working vacation $\left(P_{\mathrm{wv}}\right)$ increases, and the probability that the server is idle $\left(P_{\text {id }}\right)$ increases. It is also seen that as vacation parameter $\phi$ increases both $P_{B}$ and $P_{\text {id }}$

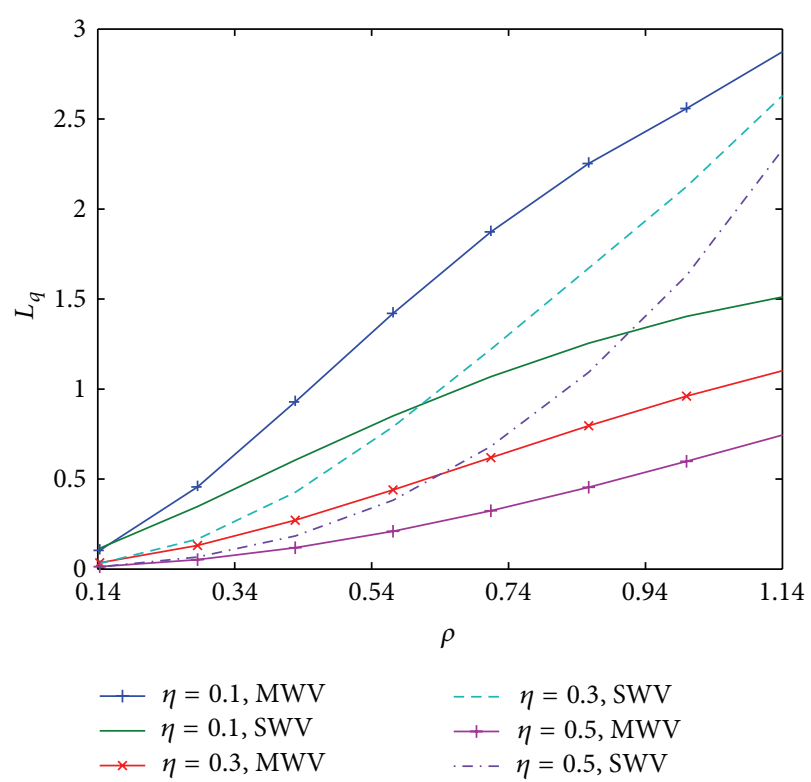

FIGURE 2: Effect of $\rho$ on $L_{q}$.

increase. But, with the increase of vacation parameter $\phi, P_{\mathrm{wv}}$ decreases.

The impact of $\eta$ on the state probabilities of the server for various values of vacation parameter $\phi$ for MWV model is shown in Figure 4. It is observed that the probability that the server is busy $\left(P_{B}\right)$ decreases, whereas the probability that the server is in working vacation $\left(P_{\mathrm{wv}}\right)$ increases as $\eta$ increases. 


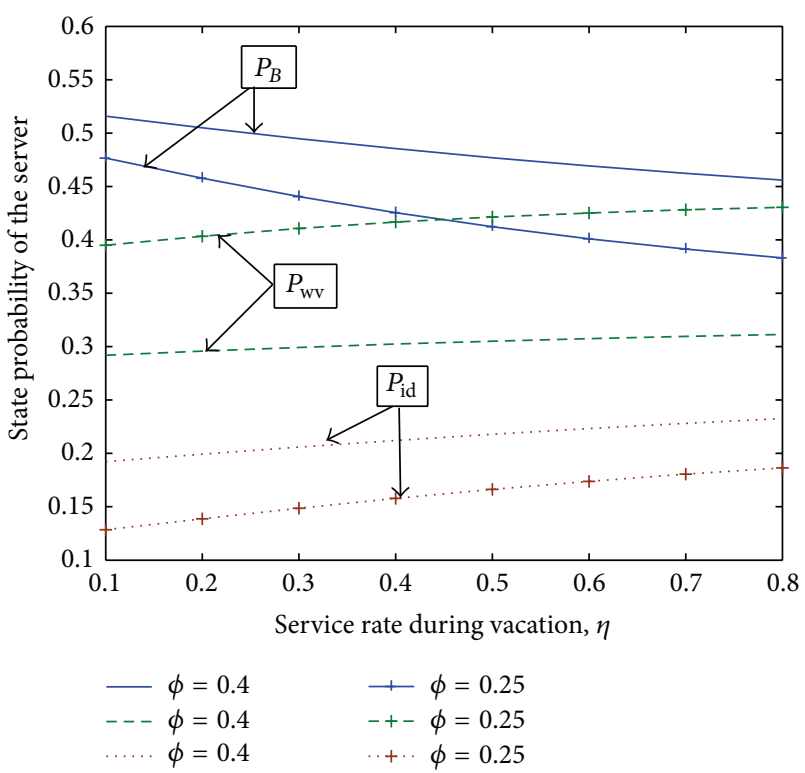

FIGURE 3: Impact of $\eta$ on state probability of the server, SWV.

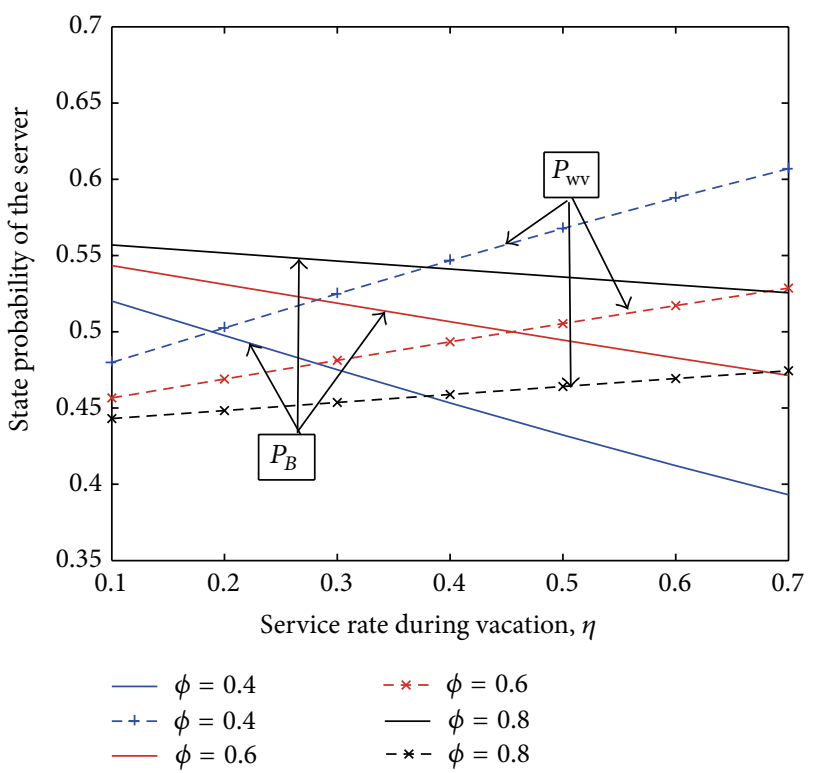

FIGURE 4: Impact of $\eta$ on state probability of the server, MWV.

As expected, $P_{B}$ increases as vacation parameter $\phi$ increases but $P_{\mathrm{wv}}$ decreases with the increase of vacation parameter $\phi$.

The impact of $\phi$ on the average rate of customer loss (L.R.) with MWV policy for different balking functions (i) $b_{i}=1-$ $\left(i / N^{2}\right)$, (ii) $b_{i}=1-(i / N)$, (iii) $b_{i}=1 /(i+1)$, and (iv) $b_{i}=1-e^{-i}$ is shown in Figure 5. It is evident from the figure that as $\phi$ increases the average rate of customer loss (L.R.) decreases for different balking functions. But the average waiting time in the system is lower for the balking function given in (i).

Figure 6 depicts the total expected cost $(F(\mu))$ as a function of regular service rate $(\mu)$ when the server is working with different service rates during vacation period for SWV

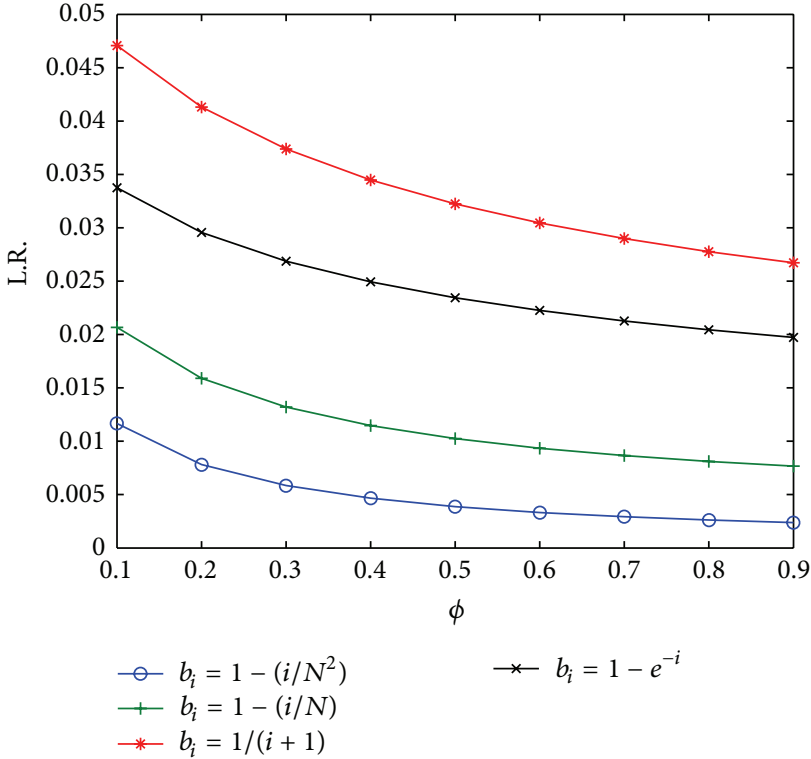

Figure 5: Impact of $\phi$ on L.R. for MWV.

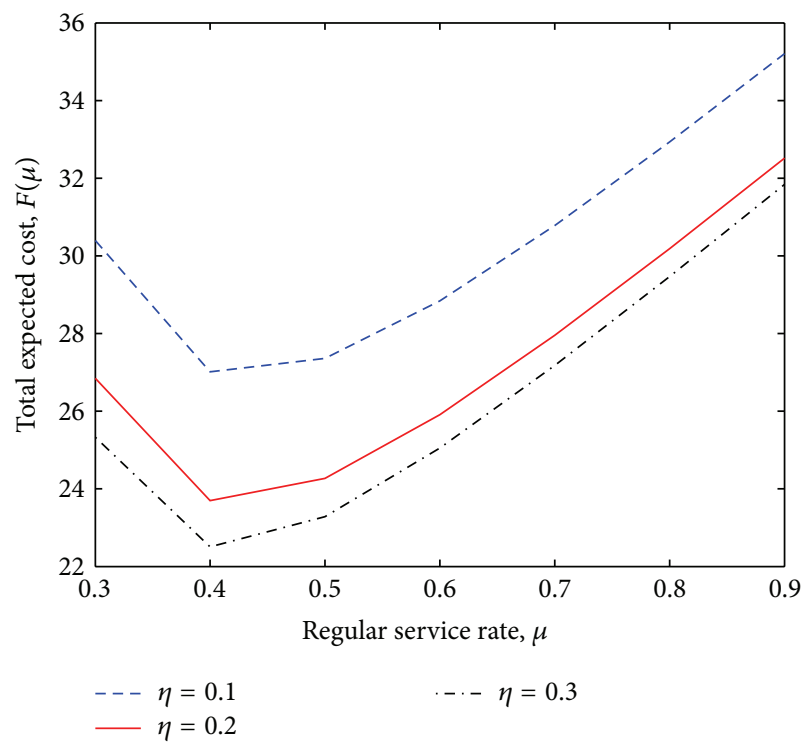

FIGURE 6: Effect of $\mu$ on total expected cost for SWV.

model. The parameters are taken as $N=10, \phi=0.1, \lambda=$ $0.2, \eta=0.1, \alpha=0.01, C_{1}=25, C_{2}=20, C_{3}=18$, and $C_{4}=15$. One may observe that for fixed $\eta$, the total expected cost decreases when service rate $\mu \leq 0.4$ and increases for $\mu>0.4$. However, when the service rate during vacation $\eta$ is increased the total expected cost reduces substantially. This infers that increasing the service rate $\mu$ beyond a certain level cannot further reduce total expected cost. These computed performance measures illustrate the performance effects of assigning job ability during the vacation period. Also the balking and reneging have a clear effect on the probabilities as we can see that there is a significant initial deviations in the state probabilities of the server. 


\section{Conclusion}

In this paper, we have carried out an analysis of balking and reneging in finite-buffer discrete-time single server queue with single and multiple working vacations for the late arrival system with delayed access from an economic viewpoint. The balking and reneging factors make our system more practical. The customers are allowed to balk or leave after joining the queue without getting served due to renege. We have developed closed-form expressions of the stationary state probabilities using recursive method. Various performance measures are presented. Numerical results in the form of tables and graphs are discussed to display the effect of the system parameters on the performance measures. The cost model proposed in our study may be useful in determining the optimal service rate needed for operating the system smoothly at optimum cost. The given model can be generalized to multiple servers with balking, reneging, and working vacations. The important significance of the findings reported in this paper is that managers of service systems can assist customers in making the right decision by providing them with precise estimates of expected time in the system. Better tradeoff of balking rate and service rate may improve decision making and satisfaction and it also lowers reneging. The modeling analysis for the discretetime queueing systems with balking, reneging, and working vacations may be applied in many congestion situations of communication systems, manufacturing systems, production/inventory systems, transportation, and many others.

\section{Conflict of Interests}

The authors declare that there is no conflict of interests regarding the publication of this paper.

\section{References}

[1] H. Takagi, Queueing Analysis-A Foundation of Performance Evaluation, vol. 3, North Holland, Amsterdam, The Netherlands, 1993.

[2] L. D. Servi and S. G. Finn, "M/M/1 queues with working vacations (M/M/1/WV)," Performance Evaluation, vol. 50, no. 1, pp. 41-52, 2002.

[3] D.-A. Wu and H. Takagi, " $M / G / 1$ queue with multiple working vacations," Performance Evaluation, vol. 63, no. 7, pp. 654-681, 2006.

[4] N. Tian, Z. Ma, and M. Liu, "The discrete time GEOm/GEOm/1 queue with multiple working vacations," Applied Mathematical Modelling, vol. 32, no. 12, pp. 2941-2953, 2008.

[5] J. Li and N. Tian, "Analysis of the discrete time Geo/Geo/1 queue with single working vacation," Quality Technology \& Quantitative Management, vol. 5, no. 1, pp. 77-89, 2008.

[6] P. Liao, "Optimal staffing policy for queueing systems with cyclic demands: waiting cost approach," in Proceedings of the 18th Annual Conference of the Production and Operations Management Society (POMS '07), Dallas, Tex, USA, 2007.

[7] D. Yue, Y. Zhang, and W. Yue, "Optimal performance analysis of an $\mathrm{M} / \mathrm{M} / \mathrm{1} / \mathrm{N}$ queue system with balking, reneging and server vacation," International Journal of Pure and Applied Mathematics, vol. 28, pp. 101-115, 2006.
[8] R. Tian and D. Yue, "Optimal balking strategies in a Markovian queue with a single vacation," Journal of Information \& Computational Science, vol. 9, no. 10, pp. 2827-2841, 2012.

[9] Y. Ma, W.-q. Liu, and J.-h. Li, "Equilibrium balking behavior in the $G e o / G e o / 1$ queueing system with multiple vacations," Applied Mathematical Modelling, vol. 37, no. 6, pp. 3861-3878, 2013.

[10] F. Zhang, J. Wang, and B. Liu, "Equilibrium balking strategies in Markovian queues with working vacations," Applied Mathematical Modelling, vol. 37, no. 16-17, pp. 8264-8282, 2013.

[11] P. Vijaya Laxmi, V. Goswami, and K. Jyothsna, "Analysis of finite buffer Markovian queue with balking, reneging and working vacations," International Journal of Strategic Decision Sciences, vol. 4, no. 1, pp. 1-23, 2013.

[12] P. V. Laxmi, V. Goswami, and K. Jyothsna, "Optimization of balking and reneging queue with vacation interruption under N-policy," Journal of Optimization, vol. 2013, Article ID 683708, 9 pages, 2013.

[13] M. Lozano and P. Moreno, "A discrete time single-server queue with balking: economic applications," Applied Economics, vol. 40, no. 6, pp. 735-748, 2008.

[14] P. Vijaya Laxmi, V. Goswami, and K. Jyothsna, "Analysis of discrete-time single server queue with balking and multiple working vacations," Quality Technology and Quantitative Management, vol. 10, no. 4, pp. 441-454, 2013.

[15] J. J. Hunter, Mathematical Techniques of Applied Probability, Discrete-time models: Techniques and applicationss and applications, Academic Press, New York, NY, USA, 1983.

[16] A. Gravey and G. Hébuterne, "Simultaneity in discrete-time single server queues with Bernoulli inputs," Performance Evaluation, vol. 14, no. 2, pp. 123-131, 1992.

[17] R. L. Rardin, Optimization in Operations Research, Prentice Hall, Upper Saddle River, NJ, USA, 1997. 


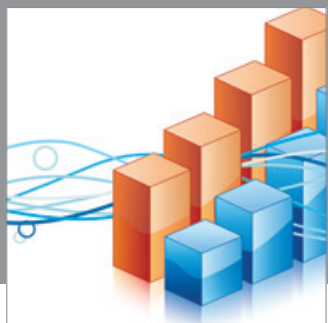

Advances in

Operations Research

mansans

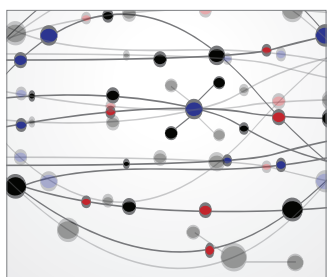

The Scientific World Journal
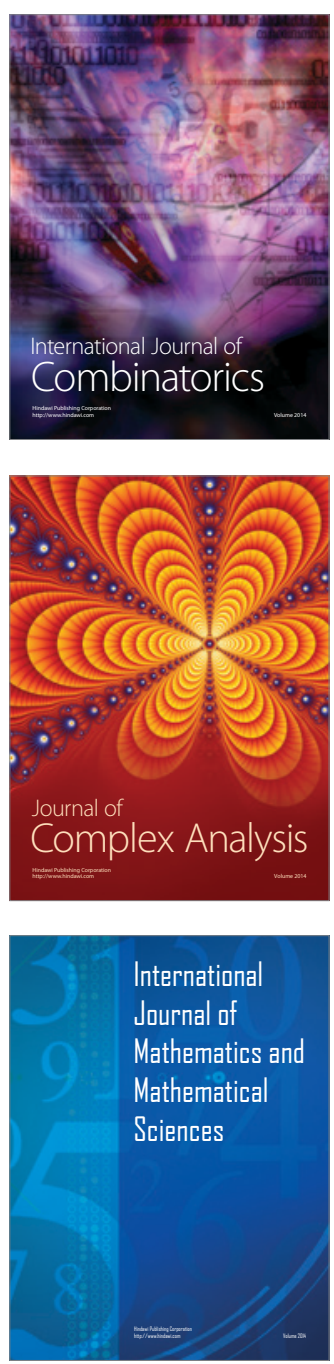
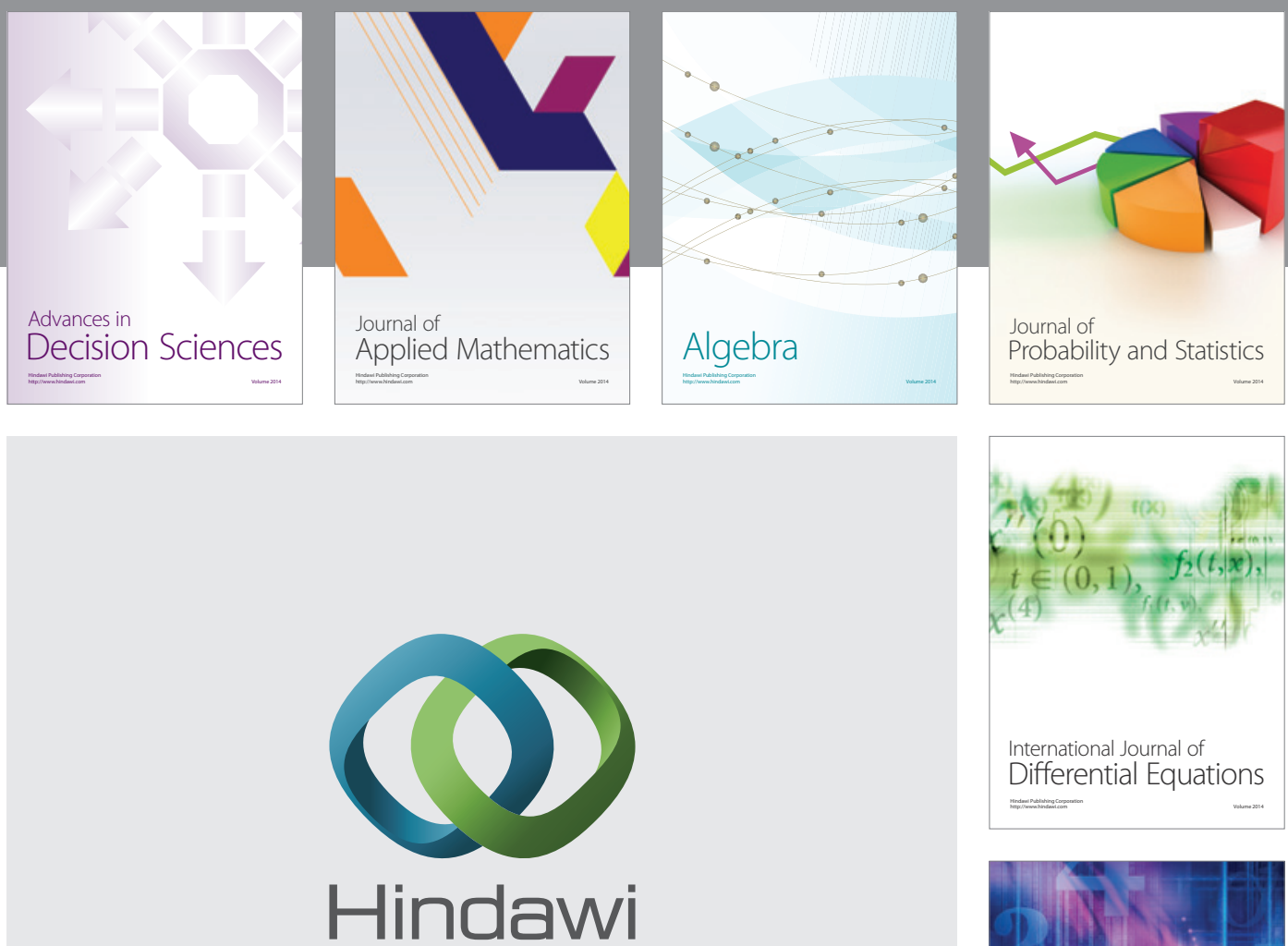

Submit your manuscripts at http://www.hindawi.com
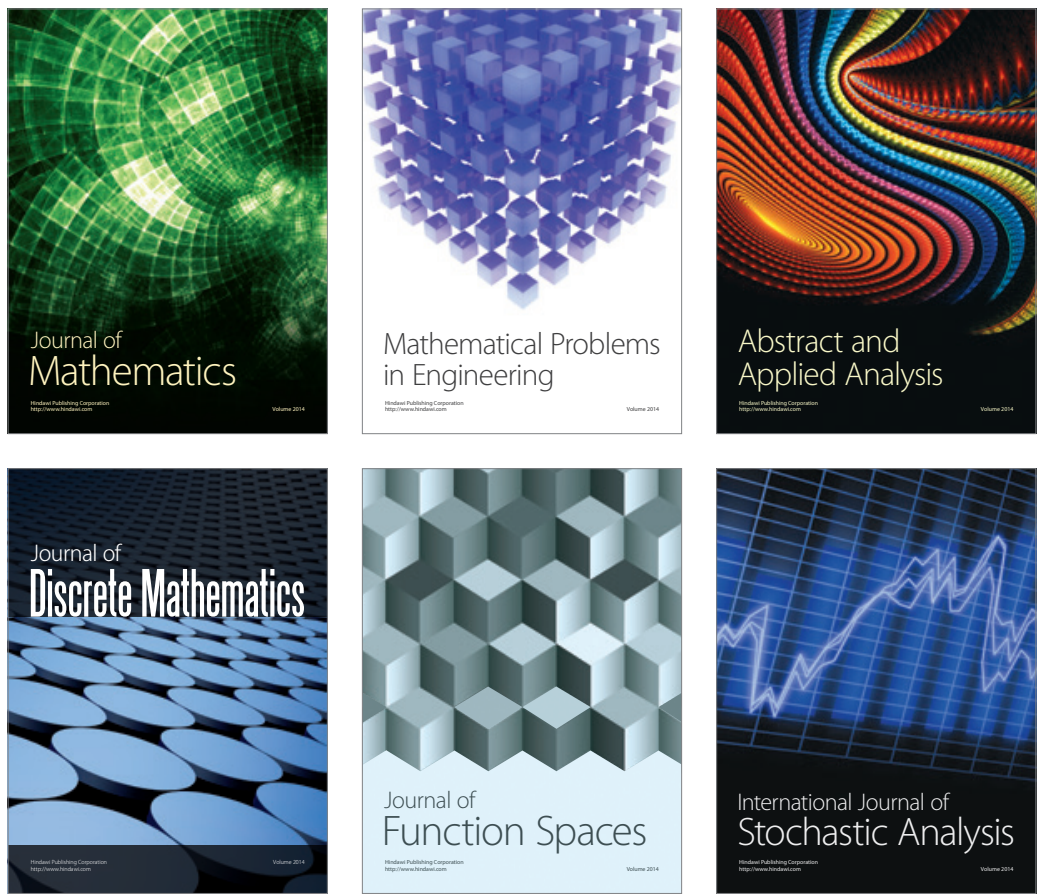

Journal of

Function Spaces

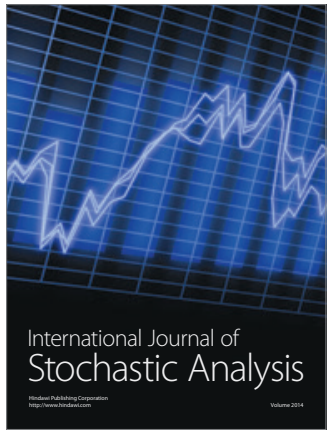

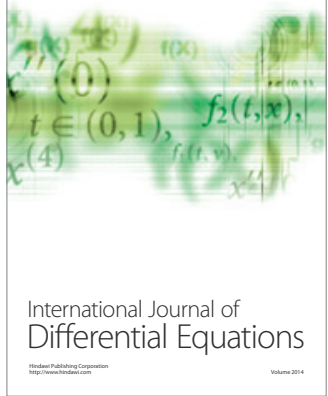
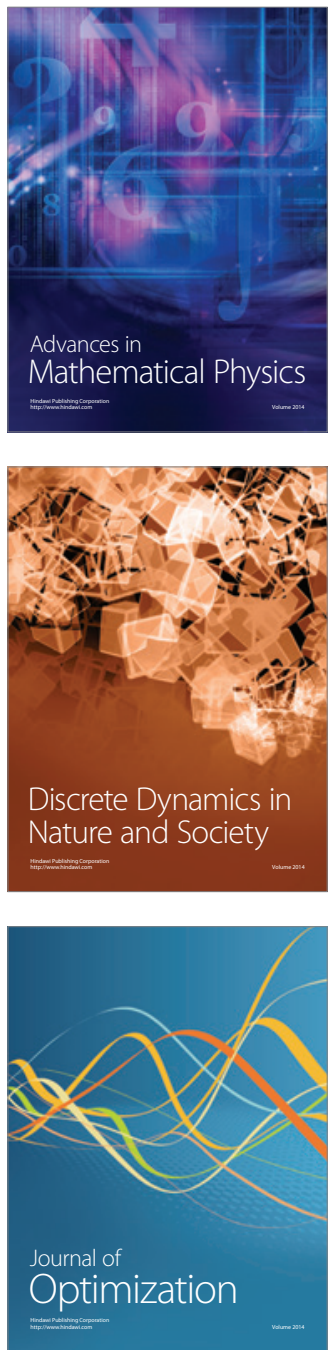